\title{
Electrochemically Induced Surface-Enhanced Infrared Difference Absorption (SEIDA) Spectroscopy of a Protein Monolayer
}

\author{
Kenichi Ataka and Joachim Heberle* \\ Forschungszentrum Jülich, IBI-2: Structural Biology, 52425 Jülich, Germany
}

Received February 13, 2003; E-mail: j.heberle@fz-juelich.de

Monolayers of proteins participating in biological electrontransfer reactions are very attractive for investigations of the structure and dynamics of intermolecular electron transfer within the native cell. Infrared (IR) spectroscopy is a very powerful method to resolve the structural dynamics of proteins on the atomic level. For the first time, we succeeded in recording infrared difference spectra of a protein monolayer by exploiting the surface enhancement exerted by a modified gold surface.

The electrochemically induced oxidation and reduction process of cytochrome $c$ (cyt $c$ ), a soluble $12.5 \mathrm{kDa}$ protein that mediates single-electron transfer between the integral membrane protein complexes of the respiratory chain, is regarded as the ideal model system for such a study. Enormous efforts have been made using classical electrochemical methods, and considerable contributions have been achieved for the understanding of the thermodynamics and kinetics of this system. ${ }^{1}$ Yet, the lack of structural information from these methods has limited the understanding of the correlation between the protein structure and the reaction kinetics. Vibrational spectroscopy, such as Raman scattering or IR absorption spectroscopy, can fill this gap. However, for the study of biomolecules at the solid/liquid interface these methods are still in their infancies. The information provided by Raman spectroscopy is mostly restricted to the chromophore due to the necessity of resonance enhancement. ${ }^{2}$ The vibrations of the whole protein are probed by Fourier transform infrared (FT-IR) spectroscopy where the functionally relevant vibrational changes are resolved by recording the reaction-induced difference. This approach has been extremely successful in the elucidation of the catalytic mechanism of many enzymes. . $^{3,4}$

Conventional IR spectroscopy suffers from sensitivity too poor to detect the minute spectral changes from a monolayer. To overcome this difficulty, we employ surface enhanced infrared absorption (SEIRA) spectroscopy. ${ }^{5}$ The rough gold surface crucial for the SEIRA effect, serves as the working electrode permitting full control of the applied voltage across the self-assembled protein monolayer. Briefly, a thin gold film ( $\sim 10 \mathrm{~nm}$ thick) is formed on a silicon hemicylinder prism (surface area: $1.5 \mathrm{~cm}^{-2}$ ) by an electroless deposition technique. ${ }^{6}$ The gold surface is exposed for $10 \mathrm{~min}$ to a $1 \mathrm{mM}$ solution of the surface modifier mercaptopropionic acid (MPA) to form a self-assembled monolayer (SAM). After rinsing the SAM with water, the prism was inserted into a spectro-electrochemical cell. The sample solution was $10 \mathrm{mM} \mathrm{K}_{2}$ $\mathrm{SO}_{4}$ and $10 \mathrm{mM}$ phosphate buffer, $\mathrm{pH} 7.0$, with $2 \mu \mathrm{M}$ horse heart cyt $c$ (Sigma). The electrode was left at the open circuit potential for $1 \mathrm{~h}$ to adsorb cyt $c$ onto the MPA layer. The adsorption kinetics were followed by continuously recording IR spectra with the attenuated total reflection (ATR)-Kretschmann configuration (modified setup from ${ }^{7}$ ), and maximum coverage was reached within 30 min. Figure 1 depicts the cyclic voltammogram of a cyt $c$ monolayer adsorbed onto the Au film electrode. Reversible oxidation and reduction currents are observed at peak positions of +55 and +9

4986 - J. AM. CHEM. SOC. 2003, 125, 4986-4987

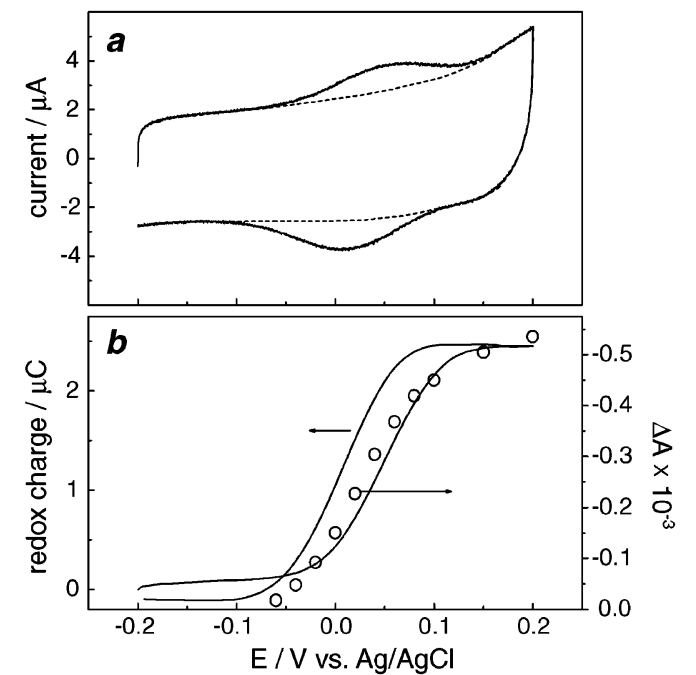

Figure 1. (a) Cyclic voltammogram of horse heart cytochrome $c$ adsorbed to the MPA modified Au electrode. Scan rate is $50 \mathrm{mV} \mathrm{s}^{-1}$. The dashed line corresponds to the double layer capacitance. (b) Total charge pass during oxidation and reduction of cytochrome $c$ (solid curve) calculated by integration of the redox current displayed in (a). Open circles are the intensities of the band at $1692 \mathrm{~cm}^{-1}$ of the difference spectra displayed in Figure 2 .

$\mathrm{mV}$, respectively. Thus, the formal potential is $+32 \mathrm{mV}$ vs $\mathrm{Ag} /$ $\mathrm{AgCl}$ (228 $\mathrm{mV}$ vs NHE), which is in the range of what is observed for cyt $c$ in solution. ${ }^{8}$ This result demonstrates that cyt $c$ is structurally and dynamically not impaired by the adherence to the SAM of MPA. The electronic charge which is transferred during a full redox reaction is $2.3 \mu \mathrm{C}$. According to Miyake et al., ${ }^{6}$ the roughness factor of the gold surface is 2.5 . This yields a surface coverage of $6.2 \times 10^{-12} \mathrm{~mol} / \mathrm{cm}^{2}$, i.e., only about half of the full coverage $\left(13.5 \times 10^{-12} \mathrm{~mol} / \mathrm{cm}^{2}\right) .{ }^{1}$ Therefore, the signals detected in the SEIRA difference spectra (Figure 2) arise from only partial coverage, demonstrating the acute sensitivity of the technique.

Figure 2 represents a series of difference spectra of cyt $c$ between the fully reduced state and varying degrees of oxidation. The reference spectrum was taken at $-0.1 \mathrm{~V}$. Then, the potential was successively increased, and sample spectra were acquired. The recorded spectra are, thus, of the oxidized-minus-reduced state. Each spectrum is an average of 5120 scans. Neither baseline correction nor any smoothing procedure has been applied. The strongest band appears at $1692 \mathrm{~cm}^{-1}$. This difference feature has been assigned to the amide $\mathrm{I}$ band (mostly $\mathrm{C}=\mathrm{O}$ stretch of the peptide bond) of a $\beta$-turn segment from the reduced form. ${ }^{5}$ The appearance of this band is indicative for a change in hydrogen bonding of this secondary structural element. The intensity of the band is plotted versus the applied potential (open circles in Figure 1b). The solid curve presents the charge derived from the integration of the redox current in Figure 1a. It is obvious that the IR intensity profile 


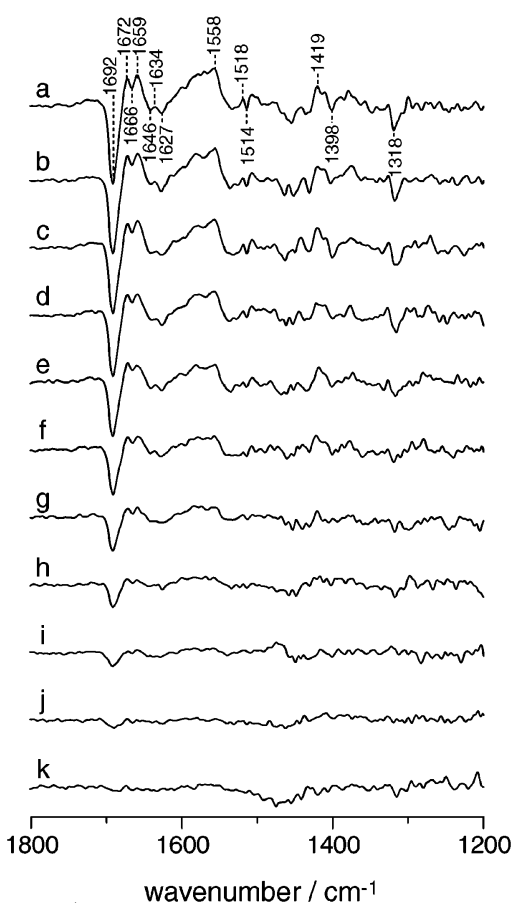

Figure 2. Redox difference spectra of cytochrome $c$ recorded with the ATR-SEIRA technique at (a) +0.20 , (b) +0.15 , (c) +0.10 , (d) +0.08 , (e) +0.06 , (f) +0.04 , (g) +0.02 , (h) 0.00 , (i) -0.02 , (j) -0.04 , (k) $-0.06 \mathrm{~V}$. The reference spectrum was taken at $-0.10 \mathrm{~V}$ vs $\mathrm{Ag} / \mathrm{AgCl}$. The band at $1692 \mathrm{~cm}^{-1}$ corresponds to an absorbance change of $-5 \times 10^{-4}$.

concurs with the electrochemical response. The formal midpoint potential derived from the spectroscopic experiment is $+32 \mathrm{mV}$. This value is identical to the value determined by cyclic voltammetry (Figure 1a). Such a strong accordance suggests that the observed absorption changes are correlated to the redox process of the adsorbed cyt $c$ monolayer (Figure 1a). Contributions from cyt $c$ molecules residing in the aqueous bulk are ruled out by the fact that the exchange to a solution free of cyt $c$, had no influence at all on the spectral response (data not shown).

Further amide I bands are observed at 1666, 1646, and 1627 $\mathrm{cm}^{-1}$ for the reduced and at 1672,1659 , and $1634 \mathrm{~cm}^{-1}$ for the oxidized state, respectively. These bands have been assigned to different types of structural changes of the protein backbone. ${ }^{9}$ Smaller bands assigned to other than amide I are observed at 1514, 1398 , and $1318 \mathrm{~cm}^{-1}$ (reduced state) and 1558, 1518, 1507, and $1419 \mathrm{~cm}^{-1}$ (oxidized state). The assignment of the positive band at $1558 \mathrm{~cm}^{-1}$ is ambiguous ${ }^{9}$ because amide II and heme modes absorb in this region. This band is partially overlapped by a broad positive feature around $1577 \mathrm{~cm}^{-1}$ which arises from the asymmetric stretching vibration of the carboxylate terminus of the MPA layer. The corresponding symmetric stretch gives rise to a small broad band peaking at $1425 \mathrm{~cm}^{-1}$. The $\mathrm{C}=\mathrm{O}$ stretch of the protonated carboxyl of MPA is hidden under the strong negative band at 1692 $\mathrm{cm}^{-1}$ of cyt $c$. These IR differences of the MPA SAM originate from the potential-induced deprotonation of the carboxylic acid group and account for the nonideal Nernstian behavior of the 1692 $\mathrm{cm}^{-1}$ band at positive voltages (see Figure 1, open circles). These findings will be addressed in a forthcoming publication.

The frequencies of the IR difference bands agree well with those reported for cyt $c$ in solution, ${ }^{9,10}$ although the relative intensities vary. Experiments with different surface modifiers indicate that the orientation of cyt $c$ along the SAM surface accounts for the variation in band intensity (data not shown). Because of the short range of the SEIRA effect $\left(<8 \mathrm{~nm}^{5}\right)$, only those vibrations are enhanced that fall within this range. Moreover, the surface selection rule of SEIRAS ${ }^{5}$ dictates that only those vibrations which involve dipole moment changes perpendicular to the local surface manifest in the IR spectrum. It has been suggested that cyt $c$ orients in a narrow angular distribution when adsorbed on a carboxyl-terminated surface. ${ }^{11}$ In this case, the surface selection has a major impact on the relative intensity of the adsorbate's band.

In conclusion, this work demonstrates that the minute enzymatic changes of a protein can be studied on the level of a monolayer. As a proof-of-principle, the electrochemically induced IR changes of a cyt $c$ adsorbed to modified gold surface have been observed with excellent signal-to-noise ratio. The surface modification preserves the full functionality of cyt $c$. The frequencies of the vibrational bands are identical to cyt $c$ in solution, whereas the observed discrepancy in relative peak intensity can be solely attributed to the characteristics of SEIRAS. The optical near-field effect of SEIRAS provides the opportunity to select those vibrators from the background of a large molecule (e.g., a protein) that are close to the gold surface. Utilization of the ATR-SEIRAS configuration is advantageous not only for increased sensitivity but also for electrochemical analysis because the presence of a bulk solution phase reduces the contribution from solution resistance as compared to that in the conventional IR technique in thin layer configuration. ${ }^{9,10}$ ATR-SEIRAS provides fast electrochemical response ${ }^{12}$ and will enable time-resolved IR studies of biological samples at solid/ liquid interfaces. Step-scan experiments ${ }^{13}$ to study the dynamics of membrane protein monolayers are currently underway. Finally, this methodology represents a nanotechnological approach toward the investigation of single native membranes and the molecular changes of their constituents upon an external trigger.

Acknowledgment. We are indebted to M. Osawa for providing details of the electroless gold deposition prior to publication and G. Büldt for continuous generous support. We kindly acknowledge the help of R. M. Nyquist for critical reading of the manuscript. This work has been supported by a grant from the Volkswagen foundation (to J.H.). K.A. is grateful to the Alexander-vonHumboldt foundation for providing a fellowship.

\section{References}

(1) Fedurco, M. Coord. Chem. Rev. 2000, 209, 263-331.

(2) Hildebrandt, P.; Murgida, D. H. Bioelectrochemistry 2002, 55, 139-143.

(3) Vogel, R.; Siebert, F. Curr. Opin. Chem. Biol. 2000, 4, 518-523.

(4) Zscherp, C.; Barth, A. Biochemistry 2001, 40, 1875-1883.

(5) Osawa, M. Bull. Chem. Soc. Jpn. 1997, 70, 2861-2880.

(6) Miyake, H.; Ye, S.; Osawa, M. Electrochem. Commun. 2002, 4, 973977.

(7) Ataka, K.; Yotsuyanagi, T.; Osawa, M. J. Phys. Chem. 1996, 100, 1066410672 .

(8) Dutton, P. L.; Wilson, D. F.; Lee, C. P. Biochemistry 1970, 9, 50775082.

(9) Schlereth, D. D.; Mäntele, W. Biochemistry 1993, 32, 1118-1126.

(10) Moss, D.; Nabedryk, E.; Breton, J.; Mäntele, W. Eur. J. Biochem. 1990 187, 565-572.

(11) Edmiston, P. L.; Lee, J. E.; Cheng, S. S.; Saavedra, S. S. J. Am. Chem. Soc. 1997, 119, 560-570.

(12) Ataka, K.; Nishina, G.; Cai, W. B.; Sun, S. G.; Osawa, M. Electrochem. Commun. 2000, 2, 417-421.

(13) Heitbrink, D.; Sigurdson, H.; Bolwien, C.; Brzezinski, P.; Heberle, J. Biophys. J. 2002, 82, 1-10.

JA0346532 